\title{
On traced monoidal closed categories
}

\author{
MASAHITO HASEGAWA \\ Research Institute for Mathematical Sciences, Kyoto University, Kyoto 606-8502, Japan \\ Email: hassei@kurims.kyoto-u.ac.jp
}

Received 28 November 2007; revised 16 May 2008

The structure theorem of Joyal, Street and Verity says that every traced monoidal category $\mathcal{C}$ arises as a monoidal full subcategory of the tortile monoidal category Int $\mathcal{C}$. In this paper we focus on a simple observation that a traced monoidal category $\mathcal{C}$ is closed if and only if the canonical inclusion from $\mathcal{C}$ into Int $\mathcal{C}$ has a right adjoint. Thus, every traced monoidal closed category arises as a monoidal co-reflexive full subcategory of a tortile monoidal category. From this, we derive a series of facts for traced models of linear logic, and some for models of fixed-point computation. To make the paper more self-contained, we also include various background results for traced monoidal categories.

\section{Introduction}

In Joyal et al. (1996), Joyal, Street and Verity introduced the notion of traced monoidal categories. They showed that every traced monoidal category $\mathcal{C}$ fully faithfully embeds in a tortile monoidal category Int $\mathcal{C}$, and that this Int-construction gives a left biadjoint of the forgetful 2-functor from the 2-category of tortile monoidal categories to that of traced monoidal categories. This remarkable result attracted much attention from theoretical computer scientists, particularly in connection with linear logic (Girard 1987) and the Geometry of Interaction (Girard 1989; Abramsky and Jagadeesan 1994; Abramsky 1996; Haghverdi 2000; Abramsky et al. 2002; Haghverdi and Scott 2006), where the special case of traced symmetric monoidal categories and compact closed categories (Kelly and Laplaza 1980) is of interest.

In this paper we shall see that the monoidal closed structure can be tied with the Int-construction in an unexpected manner. Namely, we show the following result (Theorem 4.1).

Theorem. A traced monoidal category $\mathcal{C}$ is closed if and only if the embedding from $\mathcal{C}$ into Int $\mathcal{C}$ has a right adjoint.

Despite its simplicity, to the best of our knowledge, this fact has not previously been pointed out in the literature. Perhaps this is partly because the Int-construction works too nicely: tortile monoidal categories are closed, therefore every traced monoidal category embeds into a closed one just via the Int-construction. So it seems that for this reason it was not felt that traced monoidal closed categories were themselves interesting (an exception being Coccia et al. (2002), where traced monoidal closed categories with extra structure are used for modelling higher-order cyclic shared structures). However, the tortile structure (or compact closed structure in the symmetric case) just describes a very special 
kind of closedness, and not every traced monoidal closed category is tortile. Our result says that the relation between tortile structure and general closed structure is still in harmony: every traced monoidal closed category arises as a monoidal co-reflexive full subcategory of a tortile monoidal category. The embedding from $\mathcal{C}$ to Int $\mathcal{C}$ may not preserve the closed structure (see Remark 4.2 for a counterexample), but the closed structure on $\mathcal{C}$ is determined by the closed structure of Int $\mathcal{C}$ and the co-reflection.

The author noticed this result in July 2005, after learning from Paul-André Melliès about the traced monoidal closed category of negative Conway games (Melliès 2004). It took the author a few months to realise that it had several applications to models of linear logic and to models of fixed-point computation. After that, Shin-ya Katsumata and Susumu Nishimura discovered a concrete example of this in the study of program transformations (Katsumata and Nishimura 2006), and then Katsumata gave a striking application in the theory of attribute grammars (Katsumata 2008). These discoveries prompted the author to write down the result so that it is hopefully accessible to theoretical computer scientists with less background in category theory.

To this end, instead of just stating and proving the main result (which would only need a few pages - see Section 4), we shall include in Sections 2 and 3 all the required definitions and results (but without proofs) on monoidal categories and monoidal functors, traced monoidal categories, tortile monoidal categories and Int-construction, making the paper largely self-contained. Section 5 is devoted to applications in models of linear logic and fixed-point computation; for the latter the author's old work on recursion created from cyclic sharing (Hasegawa 1997; Hasegawa 1999) is recast as linear fixed-point operators in traced models of intuitionistic linear logic. In addition, we shall make use of the graphical presentation for monoidal categories and monoidal functors (Joyal and Street 1991; Cockett and Seely 1999; Melliès 2006), which goes back to Penrose's diagrams for calculating with tensors (Penrose and Rindler 1984). This graphical approach is intuitively helpful and technically convenient, and now finding applications in computer science, see, for example, Melliès (2006) and Selinger (2007). Appendices A and B contain graphical proofs of a few background results on traced monoidal categories, and Appendix $\mathrm{C}$ gives a graphical derivation of the linear fixed-point equation.

While most work in computer science focuses on the symmetric case (traced symmetric monoidal categories and compact closed categories), in this paper, whenever possible, we consider general traced balanced (braided) monoidal categories and tortile monoidal categories, following the original development by Joyal, Street and Verity; most of our results are valid for this generality - we also expect that non-symmetric situations will become useful in future developments in computer science, possibly in the area of topological quantum computation (Freedman et al. 2002).

\section{Preliminaries}

\subsection{Monoidal categories}

A monoidal category (Mac Lane 1971) (or tensor category (Joyal and Street 1993)) $\mathcal{C}=$ $(\mathcal{C}, \otimes, I, a, l, r)$ consists of a category $\mathcal{C}$, a functor $\otimes: \mathcal{C} \times \mathcal{C} \rightarrow \mathcal{C}$, an object $I \in \mathcal{C}$ and 
natural isomorphisms $a_{A, B, C}:(A \otimes B) \otimes C \stackrel{\sim}{\rightarrow} A \otimes(B \otimes C), l_{A}: I \otimes A \stackrel{\sim}{\rightarrow} A$ and $r_{A}: A \otimes I \stackrel{\sim}{\rightarrow} A$ such that the following two diagrams commute for all $A, B, C$ and $D$ :
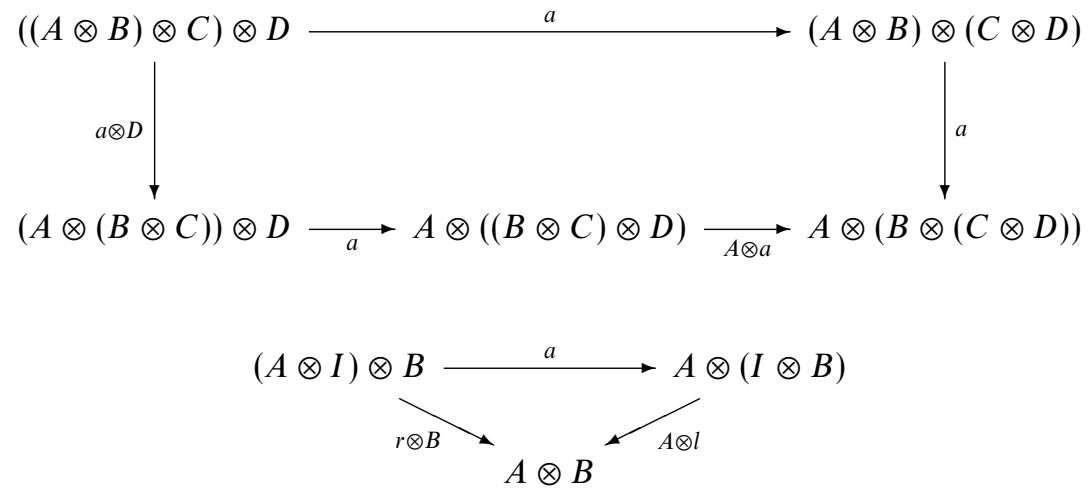

Mac Lane's coherence theorem (Mac Lane 1971; Joyal and Street 1993) states that all diagrams built from $a, l$ and $r$ commute. It follows that, in principle, most results stated for the strict monoidal categories (where $a, l, r$ are the identity arrows and $(A \otimes B) \otimes C$ is identified with $A \otimes(B \otimes C)$, and similarly for tensor unit) can be reformulated and proved without strictness. For ease of presentation, in many places in this paper we will not put brackets on tensor products when rigour is guaranteed by the coherence theorem.

A braiding is a natural isomorphism $c_{A, B}: A \otimes B \stackrel{\sim}{\rightarrow} B \otimes A$ such that both $c$ and $c^{-1}$ satisfy the following 'bilinearity' diagrams (the case for $c^{-1}$ is omitted):

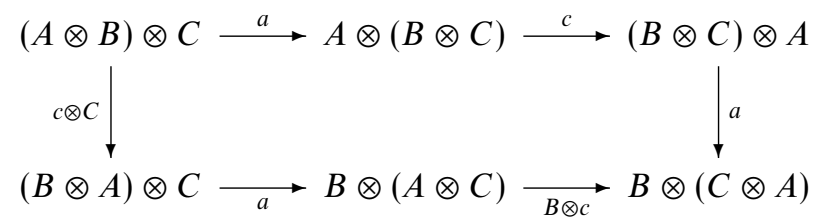

A symmetry is a braiding such that $c_{A, B}=c_{B, A}^{-1}$. A braided/symmetric monoidal category is a monoidal category equipped with a braiding/symmetry.

A twist or a balance for a braided monoidal category is a natural isomorphism $\theta_{A}$ : $A \stackrel{\sim}{\rightarrow} A$ such that $\theta_{I}=i d_{I}$ and $\theta_{A \otimes B}=c_{B, A} \circ\left(\theta_{B} \otimes \theta_{A}\right) \circ c_{A, B}$ hold. A balanced monoidal category is a braided monoidal category with a twist. Note that a symmetric monoidal category is a balanced monoidal category with $\theta_{A}=i d_{A}$ for every $A$.

\subsection{Monoidal functors and natural transformations}

For monoidal categories $\mathrm{C}=(\mathrm{C}, \otimes, I, a, l, r)$ and $\mathrm{C}^{\prime}=\left(\mathrm{C}^{\prime}, \otimes \otimes^{\prime}, I^{\prime}, a^{\prime}, l^{\prime}, r^{\prime}\right)$, a monoidal functor from $\mathcal{C}$ to $\mathcal{C}^{\prime}$ is a tuple $\left(F, m, m_{I}\right)$ where $F$ is a functor from $\mathcal{C}$ to $\mathcal{C}^{\prime}, m$ is a natural transformation from $F(-) \otimes^{\prime} F(=)$ to $F(-\otimes=)$ and $m_{I}: I^{\prime} \rightarrow F I$ is an arrow in $\mathrm{C}^{\prime}$, 
satisfying the following coherence conditions:
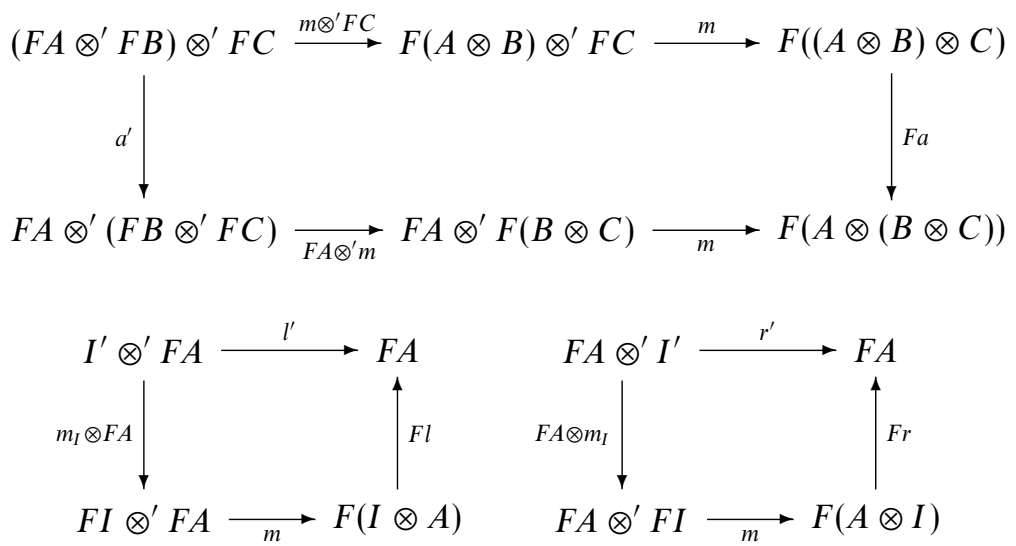

It is called strong if $m_{A, B}$ and $m_{I}$ are all isomorphisms.

A balanced monoidal functor from a balanced $\mathcal{C}$ to another $\mathcal{C}^{\prime}$ is a monoidal functor $\left(F, m, m_{I}\right)$ that additionally satisfies the condition

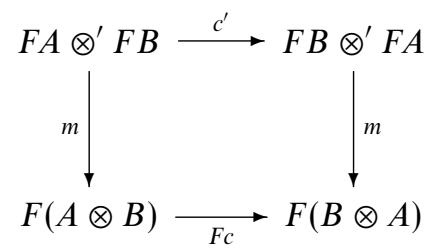

and $F\left(\theta_{A}\right)=\theta_{F A}$.

For monoidal functors $\left(F, m, m_{I}\right),\left(G, n, n_{I}\right)$ with the same source and target monoidal categories, a monoidal natural transformation from $\left(F, m, m_{I}\right)$ to $\left(G, n, n_{I}\right)$ is a natural transformation $\varphi: F \rightarrow G$ such that the following diagrams commute:
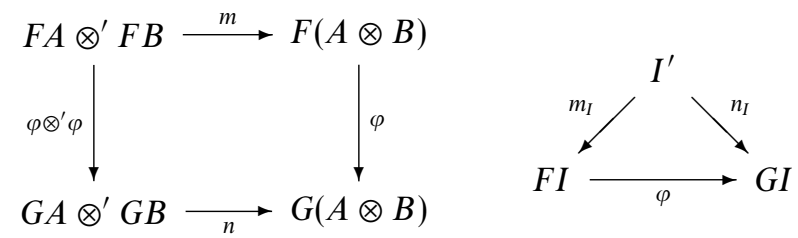

A (balanced/symmetric)monoidal adjunction between (balanced/symmetric) monoidal categories is an adjunction in which both of the functors are (balanced/symmetric) monoidal and the unit and counit are monoidal natural transformations.

\subsection{Geometry of monoidal categories}

In this paper we make use of the graphical language for monoidal categories, known as string diagrams or Penrose diagrams, that was developed by Joyal and Street (Joyal and Street 1991). A morphism $f: A_{1} \otimes A_{2} \otimes \ldots \otimes A_{m} \rightarrow B_{1} \otimes B_{2} \otimes \ldots \otimes B_{n}$ in a monoidal 
category can be drawn as (from left to right)

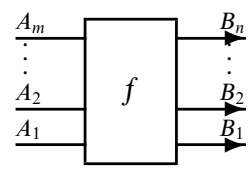

Morphisms can be composed, either sequentially,

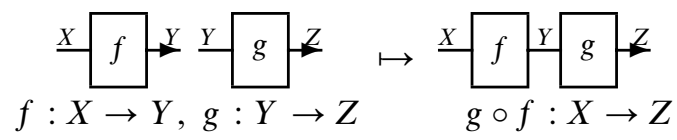

or in parallel,

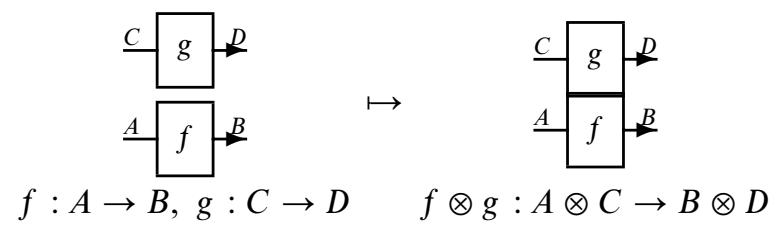

Braids are expressed by crossing:

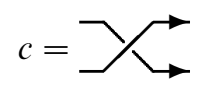

and

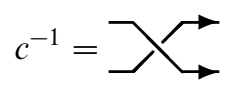

Twists are drawn as

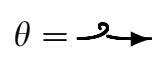

and

$$
\theta^{-1}=\stackrel{c}{\longrightarrow}
$$

Joyal and Street (1991) showed that the interpretation of such a diagram is invariant under continuous deformation, thus one may safely use graphical reasoning to establish equalities on morphisms in monoidal categories. In particular, this notation allows intuitive interpretations of the coherent conditions for braiding and twist. For example, the bilinearity axiom for a braiding can be naturally expressed as follows:

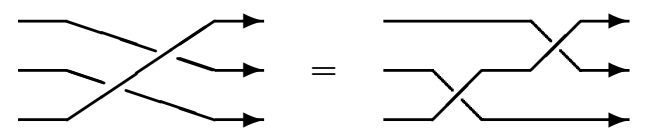

\section{CAmbridge JDURNALS}


Also, the axiom $\theta_{A \otimes B}=c_{B, A} \circ\left(\theta_{B} \otimes \theta_{A}\right) \circ c_{A, B}$ for a twist can be expressed as follows:

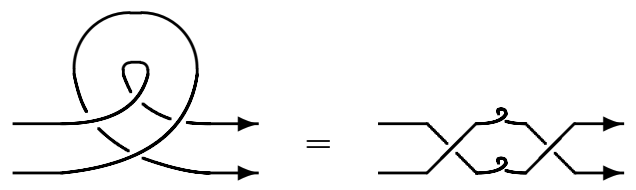

Remark 2.1. Links in these pictures should be regarded as 'ribbons' or 'framed tangles', as stressed in Joyal and Street $(1991 ; 1993)$ and Shum (1994). Our notation for twists is intended to be a reasonable alternative to the ribbon twisting notation used in the literature.

Monoidal functors and monoidal natural transformations also allow concise graphical presentations, as demonstrated by Cockett and Seely (Cockett and Seely 1999) and Melliès (Melliès 2006). Consider a monoidal functor $F=\left(F, m, m_{I}\right): \mathcal{C} \rightarrow \mathcal{D}$. Given $f: A \otimes B \rightarrow C$ in $\mathcal{C}$, we may draw a picture with a 'box' (which is drawn here with shading, and should not be confused with the square around $f$ )

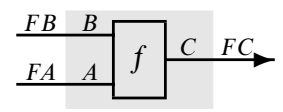

which represents $F A \otimes F B \stackrel{m_{A, B}}{\rightarrow} F(A \otimes B) \stackrel{F f}{\rightarrow} F C$. The shaded area is in $\mathcal{C}$, while the white area is in $\mathcal{D}$. Similarly, given $a: I \rightarrow A$, the picture

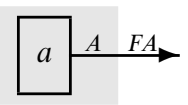

represents $I \stackrel{m_{I}}{\rightarrow} F I \stackrel{F a}{\rightarrow} F A$. The three coherence conditions of monoidal functors ensure that this notation works well for general $f: A_{1} \otimes \ldots \otimes A_{n} \rightarrow B$, where the grouping of $A_{i}$ 's does not matter. In addition, if $F$ is strong monoidal, we can do the same for tensors in the codomain. It is a pleasant exercise to write down the coherence conditions for braids and monoidal natural transformations using this box notation. For instance, one of the diagrams for monoidal natural transformations can be expressed as follows:

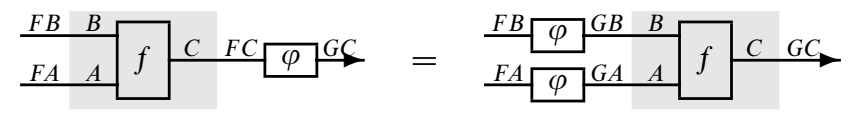

See Cockett and Seely (1999) and Melliès (2006) for further details and examples.

\section{Traced monoidal categories}

\subsection{Traced monoidal categories}

We will present a slightly simplified definition of traced monoidal categories, where trace is defined in an object-wise manner. Such a theory of object-wise trace has been developed by Blute, Cockett and Seely for linearly distributive categories (Blute et al. 2000); Milner also gave a similar axiomatisation for his reflexive action calculi (Milner 1994). 
A traced monoidal category (Joyal et al. 1996) is a balanced monoidal category $\mathcal{C}$ equipped with a family of functions, called the trace operator,

$$
\operatorname{Tr}_{A, B}^{X}: \mathcal{C}(A \otimes X, B \otimes X) \longrightarrow \mathcal{C}(A, B),
$$

subject to the following four coherence axioms, where a trace is graphically presented as a box with feedback:

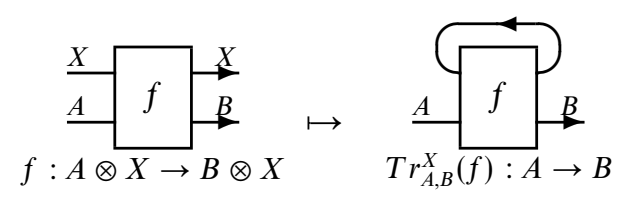

Tightening (Naturality)

$$
\operatorname{Tr}_{A^{\prime}, B^{\prime}}^{X}\left(\left(k \otimes i d_{X}\right) \circ f \circ\left(h \otimes i d_{X}\right)\right)=k \circ \operatorname{Tr}_{A, B}^{X}(f) \circ h
$$

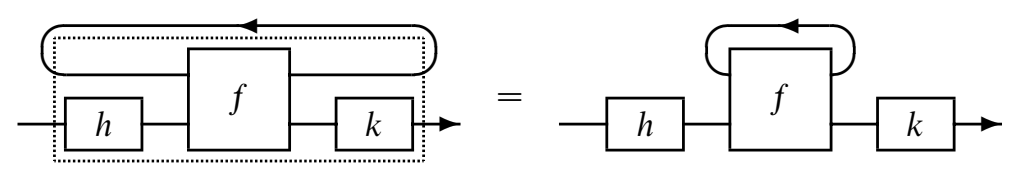

Yanking

$$
\operatorname{Tr}_{X, X}^{X}\left(c_{X, X}\right) \circ \theta_{X}^{-1}=i d_{X}=\operatorname{Tr}_{X, X}^{X}\left(c_{X, X}^{-1}\right) \circ \theta_{X}
$$

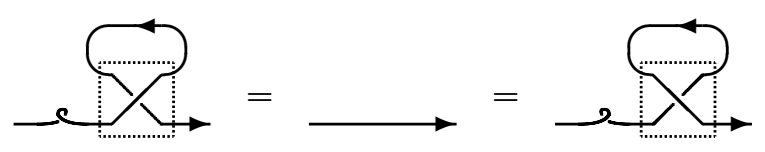

Superposing

$$
\operatorname{Tr}_{C \otimes A, C \otimes B}^{X}\left(i d_{C} \otimes f\right)=i d_{C} \otimes \operatorname{Tr}_{A, B}^{X}(f)
$$

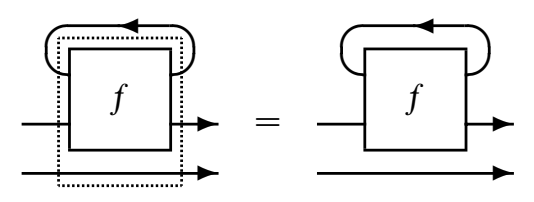

\section{CAmbridge JDURNALS}




\section{Exchange}

$$
\operatorname{Tr}_{A, B}^{X}\left(\operatorname{Tr}_{A \otimes X, B \otimes X}^{Y}(f)\right)=\operatorname{Tr}_{A, B}^{Y}\left(\operatorname{Tr}_{A \otimes Y, B \otimes Y}^{X}\left(\left(i d_{B} \otimes c_{Y, X}\right) \circ f \circ\left(i d_{A} \otimes c_{Y, X}^{-1}\right)\right)\right)
$$
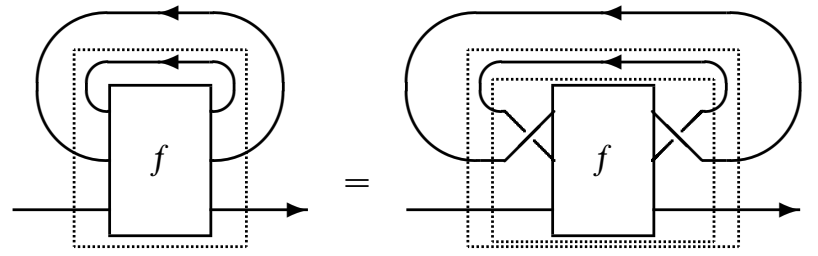

Readers familiar with the paper by Joyal, Street and Verity (Joyal et al. 1996) should find no difficulty in seeing that these are all derivable from the original axiomatisation. Conversely, the original axioms are derivable from our axioms; we shall give a slightly non-trivial derivation of Sliding and Vanishing for tensor in Appendix A. The remaining Vanishing for unit is in fact redundant in the original axiomatisation (as demonstrated in Appendix A), so our axioms are equivalent to the original axioms in ibid.

\subsection{Tortile monoidal categories}

A tortile monoidal category (Shum 1994) (which (Yetter 2001) is also called a ribbon category) is a balanced monoidal category with an object $A^{*}$ for each object $A$, unit $\eta_{A}: I \rightarrow A \otimes A^{*}$ and counit $\varepsilon_{A}: A^{*} \otimes A \rightarrow I$ such that each of

$$
\begin{gathered}
A \stackrel{l_{A}^{-1}}{\rightarrow} I \otimes A \stackrel{\eta_{A} \otimes i d_{A}}{\rightarrow}\left(A \otimes A^{*}\right) \otimes A \stackrel{a_{A, A^{*}, A}}{\rightarrow} A \otimes\left(A^{*} \otimes A\right) \stackrel{i d_{A} \otimes \varepsilon_{A}}{\rightarrow} A \otimes I \stackrel{r_{A}}{\rightarrow} A \\
A^{*} \stackrel{r_{A^{*}}^{-1}}{\rightarrow} A^{*} \otimes I \stackrel{i d_{A^{*}} \otimes \eta_{A}}{\rightarrow} A^{*} \otimes\left(A \otimes A^{*}\right) \stackrel{a_{A^{*}, A, A^{*}}}{\rightarrow}\left(A^{*} \otimes A\right) \otimes A^{*} \stackrel{\varepsilon_{A} \otimes i d_{A}}{\rightarrow} I \otimes A^{*} \stackrel{l_{A^{*}}}{\rightarrow} A^{*}
\end{gathered}
$$

is the identity and, moreover, $\theta_{A}^{*}=\theta_{A^{*}}$ holds, where, for $f: A \rightarrow B$, we have $f^{*}: B^{*} \rightarrow A^{*}$ is given by (omitting $l, r$ and $a$ )

$$
B^{*} \stackrel{i d_{B^{*}} \otimes \eta_{A}}{\rightarrow} B^{*} \otimes A \otimes A^{*} \stackrel{i d_{B^{*}} \otimes f \otimes i d_{A^{*}}}{\rightarrow} B^{*} \otimes B \otimes A^{*} \stackrel{\varepsilon_{B} \otimes i d_{A^{*}}}{\rightarrow} A^{*}
$$

It follows that $(-)^{*}$ extends to a contravariant equivalence, $A^{* *} \simeq A$, and the functor $(-) \otimes A$ is left adjoint to $(-) \otimes A^{*}$ with unit $i d_{X} \otimes \eta_{A}: X \rightarrow X \otimes A \otimes A^{*}$ and counit $i d_{X} \otimes \varepsilon_{A}: X \otimes A^{*} \otimes A \rightarrow X$. Note that tortile symmetric monoidal categories (in which braiding is a symmetry and twist is the identity) are the familiar compact closed categories (Kelly and Laplaza 1980). The unit and counit in tortile monoidal categories can be drawn as $\longrightarrow$ and $\rightarrow$, respectively. With these, the three axioms are expressed as follows:
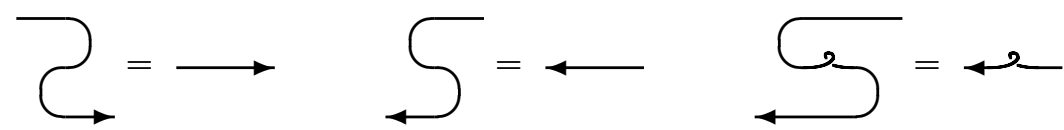

The importance of tortile monoidal categories in knot theory comes from the following result. 
Theorem 3.1 (Shum 1994). The tortile monoidal category freely generated by a single object is equivalent to the category of framed tangles.

Therefore, tortile monoidal categories give rise to invariants (or models) for tangles, in just the same sense as cartesian closed categories give rise to models of the simply typed lambda calculus (Lambek and Scott 1986).

Any tortile monoidal category has a unique trace (called the canonical trace (Joyal et al. 1996)), hence it is also a traced monoidal category. (The uniqueness of trace in tortile monoidal categories seems to be folklore; in Appendix B we shall give a direct graphical proof, based on an unpublished manuscript by the author (Hasegawa 2000).) The canonical trace is given by combining $\eta, \varepsilon, c$ and $\theta$ :

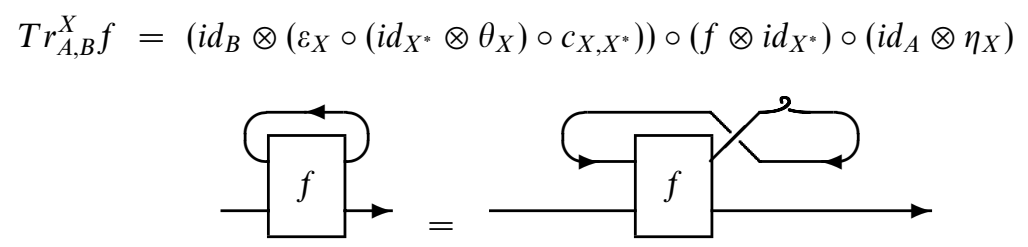

It follows that a monoidal full subcategory of a tortile monoidal category is traced.

\subsection{The Int-construction}

In fact, every traced monoidal category arises in this way: given a traced monoidal category $\mathcal{C}$, we can construct a tortile monoidal category Int $\mathcal{C}$ in which $\mathcal{C}$ fully faithfully embeds, via the Int-construction of Joyal, Street and Verity. In computer science, Int-construction can be considered as an abstract version of Girard's 'Geometry of Interaction' (Girard 1989). Abramsky introduced the GoI construction for his domaintheoretic and categorical interpretations of the Geometry of Interaction (Abramsky and Jagadeesan 1994; Abramsky 1996), which turned out to be equivalent to the symmetric case of the Int-construction. This view and its relation to Girard's work were further investigated by Abramsky, Haghverdi and Scott (Haghverdi 2000; Abramsky et al. 2002; Haghverdi and Scott 2006).

Below we recall the ingredients of the Int-construction. Objects of Int $\mathcal{C}$ are pairs of objects of $\mathcal{C}$. An arrow from $\left(A^{+}, A^{-}\right)$to $\left(B^{+}, B^{-}\right)$in $\mathbf{I n t} \mathcal{C}$ is an arrow from $A^{+} \otimes B^{-}$to $B^{+} \otimes A^{-}$in $\mathcal{C}$. The identity on $\left(A^{+}, A^{-}\right)$is $i d_{A^{+}} \otimes \theta_{A^{-}}^{-1} \in \mathcal{C}\left(A^{+} \otimes A^{-}, A^{+} \otimes A^{-}\right)$.

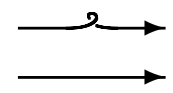

The composition of $f \in \operatorname{Int} \mathcal{C}\left(\left(A^{+}, A^{-}\right),\left(B^{+}, B^{-}\right)\right)=\mathcal{C}\left(A^{+} \otimes B^{-}, B^{+} \otimes A^{-}\right)$and $g \in$ Int $\mathrm{C}\left(\left(B^{+}, B^{-}\right),\left(C^{+}, C^{-}\right)\right)=\mathcal{C}\left(B^{+} \otimes C^{-}, C^{+} \otimes B^{-}\right)$is given by

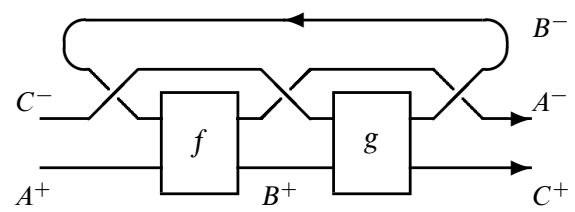


Next we look at the monoidal structure. On objects, we define tensor and unit by $\left(A^{+}, A^{-}\right) \otimes\left(B^{+}, B^{-}\right)=\left(A^{+} \otimes B^{+}, B^{-} \otimes A^{-}\right)$and $I=(I, I)$. On arrows, for $f_{1}:\left(A_{1}^{+}, A_{1}^{-}\right) \rightarrow$ $\left(B_{1}^{+}, B_{1}^{-}\right)$and $f_{2}:\left(A_{2}^{+}, A_{2}^{-}\right) \rightarrow\left(B_{2}^{+}, B_{2}^{-}\right)$, we define $f_{1} \otimes f_{2}$ by

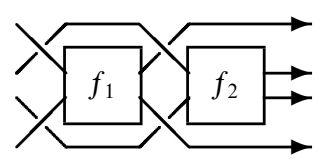

Braids and twists in Int $\mathcal{C}$ are not quite obvious:

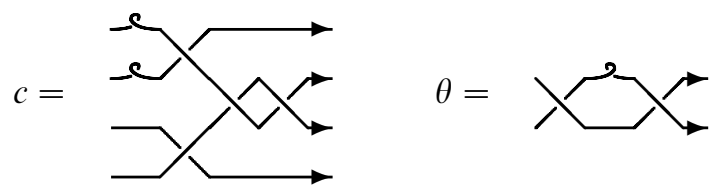

It is an interesting exercise to write down $c^{-1}$ and $\theta^{-1}$ explicitly.

Finally, we describe the duality, which is not as hard: $\left(A^{+}, A^{-}\right)^{*}=\left(A^{-}, A^{+}\right)$. The unit $\eta_{\left(A^{+}, A^{-}\right)}: I \rightarrow\left(A^{+}, A^{-}\right) \otimes\left(A^{+}, A^{-}\right)^{*}$ is given by $i d_{A^{+}} \otimes \theta_{A^{-}}^{-1}$. The counit $\varepsilon_{\left(A^{+}, A^{-}\right)}$: $\left(A^{+}, A^{-}\right)^{*} \otimes\left(A^{+}, A^{-}\right) \rightarrow I$ is $i d_{A^{-}} \otimes \theta_{A^{+}}$. We can extend $(-)^{*}$ to be a contravariant equivalence on Int $\mathcal{C}$ : on arrows $f:\left(A^{+}, A^{-}\right) \rightarrow\left(B^{+}, B^{-}\right)$, we define $f^{*}:\left(B^{+}, B^{-}\right)^{*} \rightarrow\left(A^{+}, A^{-}\right)^{*}$ as

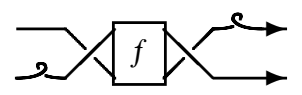

Theorem 3.2 (Joyal et al. 1996). These data determine a tortile monoidal structure on Int $\mathcal{C}$. Moreover, the functor $\mathcal{N}: \mathcal{C} \rightarrow \operatorname{Int} \mathcal{C}$ sending $A$ to $(A, I)$ strongly preserves the traced monoidal structure, and is full faithful.

Explicitly, the canonical trace on Int $\mathcal{C}$ can be given as follows. (It is not entirely obvious for the non-symmetric case.) For $f:\left(A^{+}, A^{-}\right) \otimes\left(X^{+}, X^{-}\right) \rightarrow\left(B^{+}, B^{-}\right) \otimes\left(X^{+}, X^{-}\right)$, its trace $\operatorname{Tr}^{\left(X+, X^{-}\right)} f:\left(A^{+}, A^{-}\right) \rightarrow\left(B^{+}, B^{-}\right)$is

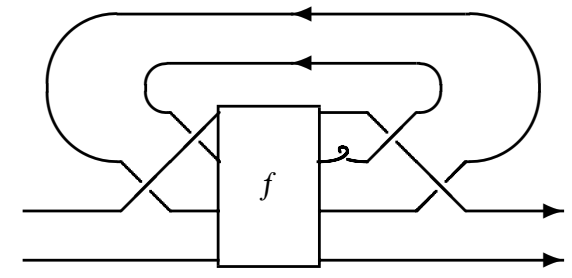

It easily follows that $\mathcal{N}$ preserves trace up to canonical isomorphisms.

In fact, Int-construction is universal, as shown in Joyal et al. (1996): it gives a left biadjoint to the forgetful 2-functor from the 2-category of tortile monoidal categories to that of traced monoidal categories. 


\subsection{Trace-fixpoint correspondence}

The following correspondence between traces and fixed-point operators on categories with finite products, which was noticed by Martin Hyland and the author independently ${ }^{\dagger}$, shows that the traces and fixed-point operators commonly used in computer science are very closely related.

Let $\mathcal{C}$ be a category with finite products. A parameterised fixed-point operator on $\mathcal{C}$ is a family of functions

$$
(-)^{\dagger}: \mathcal{C}(A \times X, X) \rightarrow \mathcal{C}(A, X)
$$

that is natural in $A$ and satisfies the fixed-point equation $f^{\dagger}=f \circ\left\langle i d_{A}, f^{\dagger}\right\rangle: A \rightarrow X$ for any $f: A \times X \rightarrow X$. It is called (Simpson and Plotkin 2000) a Conway fixed-point operator if it satisfies the dinaturality

$$
\left(f \circ\left\langle\pi_{A, X}, g\right\rangle\right)^{\dagger}=f \circ\left\langle i d_{A},\left(g \circ\left\langle\pi_{A, Y}, f\right\rangle\right)^{\dagger}\right\rangle: A \rightarrow X
$$

for $f: A \times Y \rightarrow X$ and $g: A \times X \rightarrow Y$, and the diagonal property

$$
\left(f \circ\left(i d_{A} \times \Delta_{X}\right)\right)^{\dagger}=\left(f^{\dagger}\right)^{\dagger}: A \rightarrow X
$$

for $f: A \times X \times X \rightarrow X$, where $\Delta_{X}: X \rightarrow X \times X$ is the diagonal map. (It is easy to see that dinaturality implies the fixed-point equation, so there is a redundancy in the definition.)

For readers familiar with equational theories with a fixed-point operator, it might be useful to interpret this setting as a many-sorted equational theory enriched with a fixed-point operator expressed by a $\mu$-binding operator

$$
\frac{\Gamma, x: X \vdash M: X}{\Gamma \vdash \mu x^{X} . M: X}
$$

for which the axioms above can be stated as follows (Simpson and Plotkin 2000):

$$
\begin{gathered}
\frac{\Gamma, y: Y, x: X \vdash M: X \quad \Gamma \vdash N: Y}{\Gamma \vdash\left(\mu x^{X} \cdot M\right)[y:=N]=\mu x^{X} \cdot(M[y:=N]): X} \text { naturality } \\
\frac{\Gamma, x: X \vdash M: X}{\Gamma \vdash \mu x^{X} \cdot M=M\left[x:=\mu x^{X} \cdot M\right]: X} \text { fixed-point equation } \\
\frac{\Gamma, y: Y \vdash M: X \quad \Gamma, x: X \vdash N: Y}{\Gamma \vdash \mu x^{X} \cdot M[y:=N]=M\left[y:=\mu y^{Y} \cdot N[x:=M]\right]: X} \text { dinaturality } \\
\frac{\Gamma, x_{1}: X, x_{2}: X \vdash M: X}{\Gamma \vdash \mu x_{1}^{X} \cdot \mu x_{2}^{X} \cdot M=\mu x^{X} \cdot M\left[x_{1}:=x, x_{2}:=x\right]: X} \text { diagonal property }
\end{gathered}
$$

Another equivalent axiomatisation of Conway fixed-point operators is by the Bekic property, which says that the fixed point of $f: A \times X \times Y \rightarrow X \times Y$ is equal to

$$
\left\langle\left(f_{1} \circ\left\langle i d, f_{2}^{\dagger}\right\rangle\right)^{\dagger}, f_{2}^{\dagger} \circ\left\langle i d,\left(f_{1} \circ\left\langle i d, f_{2}^{\dagger}\right\rangle\right)^{\dagger}\right\rangle\right\rangle: A \rightarrow X \times Y,
$$

\footnotetext{
$\dagger$ We should note that mathematically equivalent observations had been made by several authors before the notion of traced monoidal categories was introduced, in particular by Bloom and Ésik (Bloom and Ésik 1993) and Ştefǎnescu (Ştefǎnescu 2000).
} 
where $f_{1}=\pi \circ f: A \times X \times Y \rightarrow X$ and $f_{2}=\pi^{\prime} \circ f: A \times X \times Y \rightarrow Y$. In terms of $\mu$-binding, the Bekic property states

$$
\begin{aligned}
\Gamma, x: X, y: Y \vdash M: X \quad \Gamma, x: X, y: Y \vdash Y: N \\
\Gamma \vdash \mu\left(x^{X}, y^{Y}\right) .(M, N)=\left(\mu x^{X} . M\left[y:=\mu y^{Y} . N\right],\right. \\
\left.\mu y^{Y} . N\left[x:=\mu x^{X} . M\left[y:=\mu y^{Y} . N\right]\right]\right): X \times Y
\end{aligned}
$$

The Bekic property enables one to replace a simultaneous recursion by nested single ones, and is widely used in computer science. Many concrete fixed-point operators in computer science satisfy dinaturality and diagonal property, or, equivalently, the Bekic property, are thus Conway fixed-point operators. For example, the least fixed-point operators on various categories of domains are Conway operators.

Theorem 3.3 (Hyland, Hasegawa (Hasegawa 1997; 1999)). For any category with finite products, to give a Conway operator is to give a trace, where finite products are taken as the monoidal structure.

Here we shall just recall the constructions of this bijective correspondence:

$$
\begin{gathered}
\frac{f: A \times X \rightarrow X}{f^{\dagger}=\operatorname{Tr}_{A, X}^{X}\left(\Delta_{X} \circ f\right): A \rightarrow X} \\
\frac{g: A \times X \rightarrow B \times X}{\operatorname{Tr}_{A, B}^{X}(g)=\pi_{B, X} \circ\left(g \circ\left(i d_{A} \times \pi_{B, X}^{\prime}\right)\right)^{\dagger}: A \rightarrow B}
\end{gathered}
$$

A detailed proof can be found in Hasegawa (1999). The construction of Conway operator from a trace can be drawn as follows:

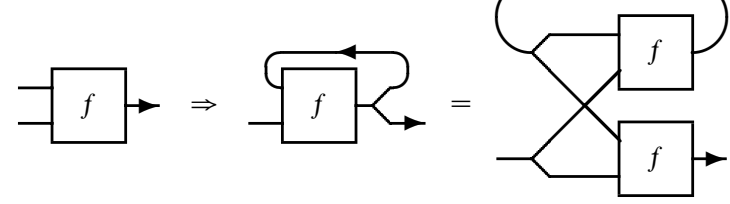

Thus, many categories in denotational semantics are traced. In particular, many categories of domains are traced, with trace determined by the least fixed-point operator. Moreover, Simpson (Simpson 1993) has shown that, under a mild condition, the least fixed-point operator is characterised as the unique dinatural fixed-point operator. Therefore, in many such domain-theoretic examples, a trace exists uniquely and is determined by the least fixed-point operator.

\section{Traced monoidal closed categories}

So far we have not thought much about closed structure, or higher-types. Recall that a monoidal category $\mathcal{C}$ is closed if $-\otimes A: \mathcal{C} \rightarrow \mathcal{C}$ has a right adjoint $A \multimap-$ for every $A$ :

$$
\mathrm{C}(X \otimes A, Y) \simeq \mathrm{e}(X, A \multimap Y) .
$$

We will denote (the $Y$-component of $)$ the counit of this adjunction by $\operatorname{ev}_{A, Y}:(A \multimap$ $Y) \otimes A \longrightarrow Y$, and for $f: X \otimes A \longrightarrow Y$, we let $\Lambda(f): X \longrightarrow A \multimap Y$ be the unique arrow satisfying $\operatorname{ev}_{A, Y} \circ\left(\Lambda(f) \otimes i d_{A}\right)=f$. 
In particular, a tortile monoidal category is closed, with $A \multimap B=B \otimes A^{*}$. The counit $\mathrm{ev}_{A, Y}: Y \otimes A^{*} \otimes A \rightarrow Y$ is given by $i d_{Y} \otimes \varepsilon_{A}$. For $f: X \otimes A \longrightarrow Y$, we have $\Lambda(f): X \longrightarrow Y \otimes A^{*}$ as $X \stackrel{i d_{X} \otimes \eta_{A}}{\longrightarrow} X \otimes A \otimes A^{*} \stackrel{f \otimes i d_{A^{*}}}{\longrightarrow} Y \otimes A^{*}$.

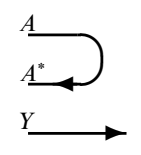

$\mathrm{ev}_{A, Y}$

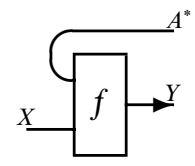

$\Lambda(f)$

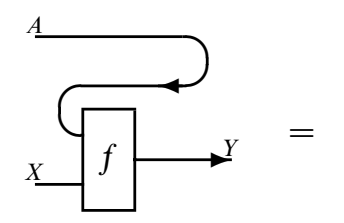

$\mathrm{ev}_{A, Y} \circ\left(\Lambda(f) \otimes i d_{A}\right)$

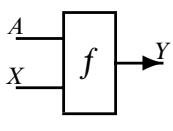

$f$

Remark 4.1. A monoidal category is said to be biclosed if, not only every $-\otimes A$ has a right adjoint $A \multimap-$, but also every $A \otimes-$ has a right adjoint $-\circ-A$. In general, a monoidal closed category may not be biclosed. Also, in a monoidal biclosed category, $A \multimap Y$ and $Y \circ A$ may not be isomorphic. However, a braided monoidal category is biclosed if it is closed, because $X \otimes A$ is naturally isomorphic to $A \otimes X$ via the braiding, and $A \multimap Y$ is isomorphic to $Y \circ A$. Therefore, when talking about traced monoidal closed categories, tortile monoidal categories, symmetric monoidal closed categories and so on, closedness automatically means biclosedness.

In the context of linear logic (Girard 1987), being symmetric monoidal closed means that we can interpret the intuitionistic multiplicative fragment (tensor $\otimes$, unit $\mathbf{1}$, and linear implication $\multimap$ ) in $\mathcal{C}$. In the rest of this paper we will see that for a traced monoidal category, closedness has yet another reading in terms of the Int-construction, which in turn is also related to the modality! and linear decomposition $A \rightarrow B=! A \multimap B$ in linear logic.

\subsection{Monoidal closed categories and co-reflection}

It is known that a monoidal co-reflective full subcategory of a monoidal closed category is also closed (although the closed structure may not be preserved by the inclusion):

Lemma 4.1 (folklore ${ }^{\dagger}$ ). Let $\stackrel{\mathcal{S}}{\stackrel{\mathcal{F}}{\perp}} \mathcal{L}$ be a monoidal adjunction. If $\mathcal{F}$ is full faithful and $\mathcal{D}$ is closed, then $\mathcal{C}$ is also closed, with $A \multimap_{\mathcal{C}} B=\mathcal{U}\left(\mathcal{F} A \multimap_{\mathcal{D}} \mathcal{F} B\right)$.

Proof.

$$
\begin{array}{rlrl}
\mathcal{C}(C \otimes A, B) & \simeq \mathcal{D}(\mathcal{F}(C \otimes A), \mathcal{F} B) & & \mathcal{F} \text { is full faithful } \\
& \simeq \mathcal{D}(\mathcal{F} C \otimes \mathcal{F} A, \mathcal{F} B) & & \mathcal{F} \text { is strong monoidal } \\
& \simeq \mathcal{D}(\mathcal{F} C, \mathcal{F} A \multimap \mathcal{F} B) & \mathcal{D} \text { is closed } \\
& \simeq \mathcal{C}(C, \mathcal{U}(\mathcal{F} A \multimap \mathcal{F} B)) \mathcal{F} \dashv \mathcal{U} .
\end{array}
$$

Here we appeal to the fact that an adjunction $\mathcal{F} \dashv \mathcal{U}$ is a monoidal adjunction if and only if $\mathcal{F}$ is strong monoidal (Kelly 1974).

\footnotetext{
$\dagger$ Martin Hyland (private communication) attributes this result to Brian Day.
} 


\subsection{Main observation}

Below we present a variation for traced monoidal categories. It characterises closedness in terms of an adjunction associated with the Int-construction.

Theorem 4.1 (main observation). Let $\mathcal{C}$ be a traced monoidal category, and $\mathcal{N}: \mathcal{C} \rightarrow$ Int $\mathcal{C}$ be the canonical inclusion from $\mathcal{C}$ into Int $\mathcal{C}$ (that is, $\mathcal{N}(A)=(A, I)$ ). Then $\mathcal{C}$ is closed if and only if $\mathcal{N}$ has a right adjoint.

Corollary 4.1. Every traced monoidal closed category is equivalent to a monoidal coreflexive full subcategory of a tortile monoidal category.

Proof of Theorem 4.1. The 'if' direction follows from the previous folklore lemma, as $\mathcal{N}$ is full faithful and strong symmetric monoidal. Note that, by the lemma,

$$
A \multimap \mathcal{C} B \simeq \mathcal{U}(\mathcal{N} A \multimap \text { Int } \mathcal{C} \mathcal{N} B)=\mathcal{U}\left((A, I)^{*} \otimes(B, I)\right) \simeq \mathcal{U}(B, A)
$$

where $\mathcal{U}$ is right adjoint to $\mathcal{N}$.

This suggests how we proceed to show the 'only if' direction. That is, if $\mathcal{C}$ is closed, define $\mathcal{U}\left(A^{+}, A^{-}\right)=A^{-} \multimap A^{+}$. For $f:\left(A^{+}, A^{-}\right) \rightarrow\left(B^{+}, B^{-}\right)$, let $U(f):\left(A^{-} \multimap A^{+}\right) \rightarrow\left(B^{-} \multimap B^{+}\right)$ be

$$
\Lambda\left(\operatorname{Tr}_{\left(A^{-} \multimap A^{+}\right) \otimes B^{-}, B^{+}}^{A^{-}}\left(f \circ\left(\operatorname{ev}_{A^{-}, A^{+}} \otimes i d_{B^{-}}\right) \circ\left(i d_{A^{-}-A^{+}} \otimes c_{B^{-}, A^{-}}\right)\right)\right)
$$

or, more internally, a map sending $k: A^{-} \rightarrow A^{+}$to $\operatorname{Tr}_{B^{-}, B^{+}}^{A^{-}}\left(f \circ\left(k \otimes B^{-}\right) \circ c_{B^{-}, A^{-}}\right): B^{-} \rightarrow B^{+}$ - see the picture

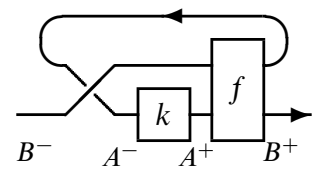

In other words, $\mathcal{U}(f)$ describes 'composition with $f$ in Int $\mathcal{C}$ ' in terms of $\mathcal{C}$.

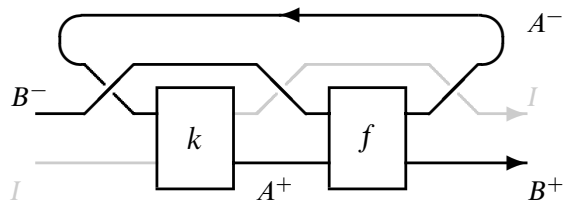

From this, it is immediate to see that $\mathcal{U}$ is indeed a functor.

Finally, it is easy to see the adjointness:

$$
\text { Int } \begin{aligned}
\mathcal{C}\left(\mathcal{N}(A),\left(B^{+}, B^{-}\right)\right) & =\operatorname{Int} \mathcal{C}\left((A, I),\left(B^{+}, B^{-}\right)\right) \\
& =\mathcal{C}\left(A \otimes B^{-}, B^{+} \otimes I\right) \\
& \simeq \mathcal{C}\left(A, B^{-} \multimap B^{+}\right) \\
& =\mathcal{C}\left(A, \mathcal{U}\left(B^{+}, B^{-}\right)\right) .
\end{aligned}
$$

The $\left(A^{+}, A^{-}\right)$-component of the counit of the adjunction is given by $\mathrm{ev}_{A^{-}, A^{+}}:\left(A^{-} \multimap\right.$ $\left.A^{+}\right) \otimes A^{-} \rightarrow A^{+}$, while the unit is trivial.

Remark 4.2. While the canonical inclusion $\mathcal{N}: \mathcal{C} \rightarrow$ Int $\mathcal{C}$ preserves the traced monoidal structure (as noted in Section 3.3), $\mathcal{N}$ may not preserve the closed 
structure: $\mathcal{N}(A \multimap B)=(A \multimap B, I)$ does not have to be isomorphic to $\mathcal{N}(B) \otimes \mathcal{N}(A)^{*} \simeq$ $(B, A)$. For example, if $\mathcal{C}$ is a traced cartesian closed category, $\mathcal{N}(A \Rightarrow 1) \simeq(1,1)$ while $\mathcal{N}(1) \otimes \mathcal{N}(A)^{*} \simeq(1, A)$, thus $\mathcal{N}$ preserves the closed structure only when every object of $\mathcal{C}$ is isomorphic to 1 .

Note that the adjunction in the theorem above gives rise to an idempotent balanced monoidal comonad $\mathcal{N U}$ on Int $\mathcal{C}$ that sends $\left(A^{+}, A^{-}\right)$to $\left(A^{-} \multimap A^{+}, I\right)$. Recall that for an idempotent comonad $K$ on a category $\mathcal{C}$, its co-Kleisli category $\mathcal{C}_{K}$ and co-EilenbergMoore category $\mathcal{C}^{K}$ are both equivalent to the co-reflexive full subcategory of $\mathcal{C}$ whose objects are in the image of $K$. Thus we have the following corollary.

Corollary 4.2. For any traced monoidal closed category $\mathcal{C}$, there is an idempotent balanced monoidal comonad on $\mathbf{I n t} \mathcal{C}$ such that its co-Kleisli category is equivalent to $\mathcal{C}$.

Thus all traced monoidal closed categories come from tortile monoidal categories with an idempotent balanced monoidal comonad. The converse is not true, however: there is a tortile monoidal category $y$ with an idempotent balanced monoidal comonad ! such that Int $\left(y_{!}\right)$is not equivalent to $y$ - see Section 6.2.

Remark 4.3. It might be the case that Int-construction and co-Kleisli construction give rise to a biadjunction between the 2-category of traced monoidal closed categories and a suitable 2-category of tortile monoidal categories with idempotent balanced monoidal comonad, but we do not know the answer yet.

\section{Applications}

\subsection{Models of linear logic}

We have already noted that symmetric monoidal closed categories are the models of multiplicative fragment of intuitionistic linear logic (IMLL). Here we shall quickly recall additional structures needed for modelling other elements of linear logic (Seely 1989; Barr 1991; Benton 1995; Bierman 1995; Barber and Plotkin 1997; Hyland and Schalk 2003; Melliès 2003).

A symmetric monoidal closed category with an object $\perp$ such that the canonical map from $A$ to $(A \multimap \perp) \multimap \perp$ is invertible for all objects $A$ is called a *-autonomous category (Barr 1991). *-autonomous categories are the models of the multiplicative fragment of classical linear logic (MLL), where $\perp$ (called a dualising object) models falsity and $A \multimap \perp$ the linear negation $A^{\perp}$ of $A$. Compact closed categories (= tortile symmetric monoidal categories) are special instances of $*$-autonomous categories, so they also are models of MLL - although they are rather degenerate ones, in that interpretations of $A$ \& $B=\left(A^{\perp} \otimes B^{\perp}\right)^{\perp}$ and $A \otimes B$ are isomorphic, and so are $\perp$ and $\mathbf{1}$.

For interpreting additive conjunctions and disjunctions of linear logic, it is enough simply to assume finite products and finite coproducts. However, they are not particularly 
important in this paper; we do not know any useful conditions to ensure the existence of finite (bi)products ${ }^{\dagger}$ on Int $\mathcal{C}$, so additives do not have a good place in our story yet.

A symmetric monoidal adjunction between a category with finite products and a symmetric monoidal category gives rise to a comonad on the symmetric monoidal category, which models the exponential ! of linear logic (Barber and Plotkin 1997; Bierman 1995). Such a comonad is called a linear exponential comonad (Hyland and Schalk 2003). Explicitly, a linear exponential comonad is a symmetric monoidal comonad ! on a symmetric monoidal category $\mathcal{C}$ such that the category of its coalgebras is a category of commutative comonoids, which means:

— there are specified monoidal natural transformations $e_{A}: ! A \rightarrow I$ and $d_{A}: ! A \rightarrow ! A \otimes ! A$ that form a commutative comonoid $\left(! A, e_{A}, d_{A}\right)$ in $\mathcal{C}$ and also are coalgebra morphisms from $\left(! A, \delta_{A}\right)$ to $\left(I, m_{I}\right)$ and $\left(! A \otimes ! A, m_{! A, ! A} \circ\left(\delta_{A} \otimes \delta_{A}\right)\right)$, respectively; and

- any coalgebra morphism from $\left(! A, \delta_{A}\right)$ to $\left(! B, \delta_{B}\right)$ is also a comonoid morphism from $\left(! A, e_{A}, d_{A}\right)$ to $\left(! B, e_{B}, d_{B}\right)$.

In summary, a model of the multiplicative exponential fragment of intuitionistic linear logic (IMELL) is a symmetric monoidal closed category with a linear exponential comonad. A model of the multiplicative exponential fragment of classical linear logic (MELL) is a *-autonomous category with a linear exponential comonad.

Returning to our study of traced categories, if $\mathcal{C}$ is a traced cartesian closed category, our main theorem implies that $\mathcal{C} \underset{\mathcal{U}}{\stackrel{\mathcal{N}}{\perp}}$ Int $\mathcal{C}$ is a symmetric monoidal adjunction, thus $\mathcal{N} U$ is a linear exponential comonad on Int $\mathcal{C}$.

Corollary 5.1. For any traced cartesian closed category $\mathcal{C}$, there is an idempotent linear exponential comonad on $\mathbf{I n t} \mathcal{C}$ such that its co-Kleisli category is equivalent to $\mathcal{C}$.

Explicitly, this comonad sends $\left(A^{+}, A^{-}\right)$to $\left(A^{-} \Rightarrow A^{+}, 1\right)$.

Taking this together with the trace-fixpoint correspondence, we have the following corollary.

Corollary 5.2. Any cartesian closed category with a Conway fixed-point operator is equivalent to one arising from a compact closed model of MELL via the co-Kleisli construction.

Remark 5.1. Given a traced symmetric category with an idempotent linear exponential comonad, its co-Kleisli category (equivalently, co-Eilenberg-Moore category) has finite products and is traced (with cartesian product as monoidal product), and hence has a Conway fixed-point operator - see Melliès (2006) for a proof. Our results above show that the Int-construction provides many such examples.

\footnotetext{
$\dagger$ If a tortile monoidal category has finite products, they are biproducts (Houston 2008). Therefore, in a degenerate model of linear logic in which multiplicative conjunction and disjunction are isomorphic (that is, a compact closed category), additive conjunction and disjunction also are isomorphic.
} 
We can go further. Since monoidal adjunctions are closed under composition, Intconstruction actually sends a traced model for IMELL to a compact closed model for MELL.

Corollary 5.3. Let $\mathcal{C}$ be a traced symmetric monoidal closed category with a linear exponential comonad ! (that is, a model of IMELL). Then Int $\mathcal{C}$ is equipped with a linear exponential comonad !' given by $!^{\prime}\left(A^{+}, A^{-}\right)=\mathcal{N}\left(!\left(\mathcal{U}\left(A^{+}, A^{-}\right)\right)\right)=\left(!\left(A^{-} \multimap A^{+}\right), I\right)$.

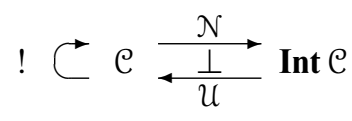

This gives an alternative possibility for interpreting exponentials in the Geometry of Interaction - this is not quite the same as Girard's (Girard 1989) or the treatment by Abramsky, Haghverdi, Scott et al. (Abramsky and Jagadeesan 1994; Haghverdi 2000; Abramsky et al. 2002; Haghverdi and Scott 2006).

\subsection{Linear fixed-points: recursion from cyclic sharing revisited}

The following result is shown in Hasegawa (1997; 1999).

Theorem 5.1. Given a symmetric monoidal adjunction $\mathcal{C} \underset{\stackrel{L}{L}}{\stackrel{F}{\mathcal{D}}}$ between a category $\mathcal{C}$ with finite products (taken as the monoidal structure) and a traced symmetric monoidal category $\mathcal{D}$, there exists a family of functions

$$
(-)^{\dagger}: \mathcal{D}(F A \otimes X, X) \longrightarrow \mathcal{D}(F A, X)
$$

that is natural in $A$ and dinatural in $X$.

Explicitly, $f^{\dagger}$ is given by

$$
\varepsilon_{X} \circ \operatorname{Tr}_{F A, F U X}^{F U X}\left(m_{U X, U X}^{F}{ }^{-1} \circ F\left(\Delta_{U X} \circ U f \circ m_{F A, X}^{U} \circ\left(\eta_{A} \times i d_{U X}\right)\right) \circ m_{A, U X}^{F}\right)
$$

where $\eta$ and $\varepsilon$ are the unit and counit of the adjunction (these should not be confused with those for tortile monoidal categories). In particular, $f^{\dagger}$ satisfies the fixed-point equation

$$
f^{\dagger}=f \circ\left(i d_{F A} \otimes f^{\dagger}\right) \circ m^{F^{-1}} \circ F \Delta_{A} .
$$

This result has been used to provide a semantics of recursion in lambda calculi with cyclic sharing (Hasegawa 1997; Hasegawa 1999).

Using the box notation (Cockett and Seely 1999; Melliès 2006), this $f^{\dagger}$ can be nicely expressed as follows:

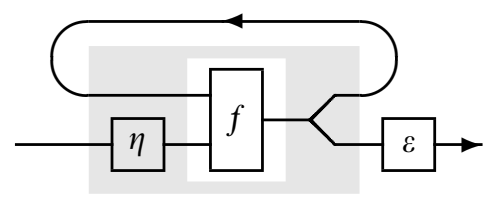


In this picture, the inner and outer boxes correspond to functors $U$ and $F$, respectively, and the components in the gray zone belong to $\mathcal{C}$ while those in white to $\mathcal{D}$. Happily, we do not have to bother with the coherence morphisms $m^{F}$ and $m^{U}$. In Appendix $\mathrm{C}$ we give a graphical derivation of the fixed-point equation using the box notation, which replaces the lengthy calculation in Hasegawa (1999).

Remark 5.2. In Hasegawa (1997; 1999), only Kleisli adjunctions of commutative monads were considered, and this theorem was stated with an additional condition that $F$ is an identity-on-object, strict monoidal functor. However, this restriction is not essential, as we demonstrate here.

If $\mathcal{D}$ is closed, the operator $(-)^{\dagger}$ can be replaced by a family of arrows of

$$
\mathcal{D}(F U(X \multimap X), X) \simeq \mathcal{D}(I, F U(X \multimap X) \multimap X) .
$$

In terms of the linear lambda calculus corresponding to intuitionistic linear logic (Barber and Plotkin 1997), this amounts to a linear fixed-point combinator $Y_{X}: !(X \multimap X) \multimap X$ such that

$$
\mathrm{Y}_{X}(! M)=M\left(\mathrm{Y}_{X}(! M)\right)
$$

holds for any term $M: X \multimap X$ with no free linear variable. Note that this is different from the usual fixed-point combinator with type ! (!X $\multimap X) \multimap X$, which returns a fixed point of a non-linear map of type $! X \multimap X$. As demonstrated in Hasegawa $(1997 ; 1999)$, linear fixed-point operators can exist even in the settings where such a standard non-linear fixed-point operator is not available ${ }^{\dagger}$.

Proposition 5.1. Suppose that $\mathcal{D}$ is a traced symmetric monoidal closed category with a linear exponential comonad!. Then there exists a family of arrows fix $\mathrm{f}_{X}: !(X \multimap X) \longrightarrow X$ such that, for any $f: ! A \otimes X \longrightarrow X$, we have $f^{\dagger}=\operatorname{fix}_{X} \circ !(\Lambda(f)) \circ \delta_{A}: ! A \rightarrow X$ (with $\Lambda(f): ! A \longrightarrow X \multimap X$ the adjoint mate of $f)$ is a fixed-point of $f$, that is, $f^{\dagger}$ agrees with

$$
! A \stackrel{d}{\rightarrow} ! A \otimes ! A \stackrel{i d_{:} \otimes f^{\dagger}}{\rightarrow} ! A \otimes X \stackrel{f}{\rightarrow} X .
$$

As we have seen, if $\mathcal{C}$ is a traced symmetric monoidal closed category with a linear exponential comonad ! (traced model of IMELL), then Int $\mathcal{C}$ is a compact closed category with a linear exponential comonad $!^{\prime}\left(A^{+}, A^{-}\right)=\left(!\left(A^{-} \multimap A^{+}\right), I\right)$ (compact model of MELL). Therefore, both $\mathcal{C}$ and Int $\mathcal{C}$ admit interpretations of such linear fixed-points. The linear fixed-point on $\left(X^{+}, X^{-}\right)$in Int $\mathcal{C}$ is determined by the linear fixed-point on $X^{-} \multimap X^{+}$in $\mathcal{C}$. Spelling out the detail, to give a linear fixed-point

$$
\mathrm{Y}_{\left(X^{+}, X^{-}\right)}: !^{\prime}\left(\left(X^{+}, X^{-}\right) \multimap\left(X^{+}, X^{-}\right)\right) \multimap\left(X^{+}, X^{-}\right)
$$

$\dagger$ One may define $Y^{\prime} X: !(! X \multimap X) \multimap X$ from $Y$ by (using the syntax of DILL (Barber and Plotkin 1997))

$$
\lambda g^{!(! X \multimap X)} \text {.let } ! f^{! X \multimap X} \text { be } g \text { in let } ! z^{X} \text { be } \mathrm{Y}_{! X}\left(\lambda y^{! X} \text {.let } ! x^{X} \text { be } y \text { in } !(f(! x))\right) \text { in } z,
$$

but this does not satisfy the non-linear fixed-point equation $\mathrm{Y}^{\prime}(! M)=M\left(!\left(\mathrm{Y}^{\prime}(! M)\right)\right)$. More concretely, consider the compact closed category Rel of sets and relations, with the powerset comonad as the linear exponential comonad - this has a linear fixed-point operator (Hasegawa 1997; 1999) but no non-linear one (cf. Melliès (2006)). 
in Int $\mathcal{C}$ is to give a map of

$$
\left(X^{-} \otimes !\left(\left(X^{+} \otimes X^{-}\right) \multimap\left(X^{+} \otimes X^{-}\right)\right)\right) \multimap X^{*}
$$

in $\mathcal{C}$, which is described as

$$
\lambda\left(y^{X^{1}} \otimes ! f^{\left(\left(X^{+} \otimes X^{-}\right) \multimap\left(X^{+} \otimes X^{-}\right)\right)}\right) \cdot Y_{X^{-} \multimap X^{+}}(!(\mathcal{U} f)) y .
$$

Example 5.1. Let $\mathcal{C}$ be a traced cartesian closed category. Take a morphism $f=\left\langle f^{+}, f^{-}\right\rangle$: $\left(X^{+}, X^{-}\right) \rightarrow\left(X^{+}, X^{-}\right)$in Int $\mathcal{C}$, thus $f^{+}: X^{+} \times X^{-} \rightarrow X^{+}$and $f^{-}: X^{+} \times X^{-} \rightarrow X^{-}$in $\mathcal{C}$. Using the simply typed lambda calculus with $\mu$-fixpoint operator as an internal language of $\mathcal{C}, \mathcal{U} f: X^{+^{X^{-}}} \rightarrow X^{+^{X^{-}}}$can be described as

$$
\lambda k^{X^{-} \rightarrow X^{+}} \cdot \lambda y^{X^{-}} \cdot f^{+}\left(\mu x^{X^{+}} \cdot k\left(f^{-}(x, y)\right), y^{-}\right):\left(X^{-} \rightarrow X^{+}\right) \rightarrow\left(X^{-} \rightarrow X^{+}\right)
$$

where $\mu z . g(z)$ denotes the corresponding Conway fixed-point. The linear fixed-point $f^{\dagger}:(1,1) \rightarrow\left(X^{+}, X^{-}\right)$is determined by a morphism from $X^{-}$to $X^{+}$in $\mathcal{C}$, which is given by the fixed-point of $\mathcal{U} f$, that is,

$$
\mu k^{X^{-} \rightarrow X^{+}} \cdot \lambda y^{X^{-}} . f^{+}\left(\mu x^{X^{+}} \cdot k\left(f^{-}(x, y)\right), y^{-}\right): X^{-} \rightarrow X^{+} .
$$

\section{Related work and discussion}

\subsection{Program transformations and attribute grammars}

Recently, Katsumata and Nishimura (Katsumata and Nishimura 2006) introduced a program transformation technique called (semantic) higher-order removal. Roughly speaking, their technique transforms a higher-order map $g:\left(A^{-} \Rightarrow A^{+}\right) \Rightarrow\left(B^{-} \Rightarrow B^{+}\right)$ (created in the process of dealing with fusions of functions with accumulating parameters, which involves certain bi-directional information flow) to a less-expensive first-order map $f: A^{+} \times B^{-} \Rightarrow B^{+} \times A^{-}$such that $\mathcal{U}(f)=g$ holds, where $\mathcal{U}$ is right adjoint to $\mathcal{N}$. They give a syntactic condition that ensures that $g$ is in the image of $\mathcal{U}$ in their semantic models, and presented a procedure for identifying $f$ such that $\mathcal{U}(f)=g$.

More recently, Katsumata (Katsumata 2008) has shown that a substantial part of the theory of attribute grammars (Knuth 1968) can be carried out very cleanly in terms of traced monoidal categories and Int-construction. Very roughly, an attribute grammar assigns computation with bidirectional information flow to term trees of a context free grammar, which can be interpreted in traced monoidal categories in just the same way as for the Geometry of Interaction:

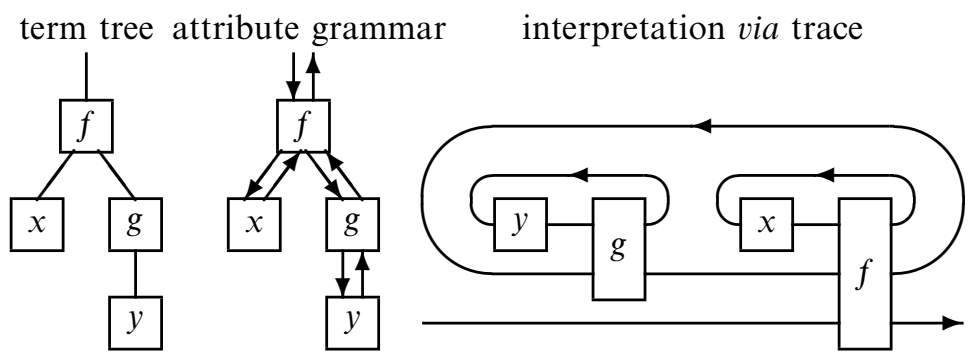

\section{CAMBridge JDURALS}


In Katsumata's work, the adjunction $\mathcal{N} \dashv \mathcal{U}$ of Theorem 4.1 provides the equivalence between attribute grammars and synthesised attribute grammars (attribute grammars with just bottom-up information flow). This generalises the result on the equivalence between such attribute grammars formulated in a domain-theoretic setting (Chirica and Martin 1979).

\subsection{Game semantics}

An interesting (non)example is the category $y$ - of negative Conway games (Melliès 2004). $y$ - is a symmetric monoidal full subcategory of the compact closed category $y$ of Conway games (Joyal 1977). The inclusion from $y^{-}$to $y$ has a right adjoint. Thus (by the folklore lemma) $y-$ is a traced symmetric monoidal closed category. $y-$ is one of very few interesting traced symmetric monoidal closed categories that are neither cartesian closed nor compact closed.

Int $y$ - is not equivalent to $y$ - thus it does not really fit in our result. But the difference is subtle; more precisely, Int $y^{-}$is equivalent to a compact closed full subcategory of $y$, whose objects are the tensor products of positive and negative games. We expect that a similar situation should be found in many categories of games, which would deserve further study. A recent relevant example is the category of multi-bracketed games of Melliès and Tabareau (Melliès and Tabareau 2007).

\subsection{Concluding remarks}

We have observed that closedness for a traced monoidal category is equivalent to an adjointness associated with the Int-construction. This simple result has a number of applications to models of linear logic and fixed-point computation. We hope that these provide some good motivation for studying traced monoidal closed categories. In particular, we expect that it is possible to develop the 'Geometry of Higher-Order Interaction' along the line of this work.

We shall conclude this paper by suggesting some further research directions. First, to obtain a more solid understanding of traced monoidal closed categories, it is desirable to find a good concrete description of free traced monoidal closed categories. Intuitively, it should be some sort of 'higher-order tangles' - in the same sense that a free traced monoidal category can be given as a category of the usual (framed) tangles. Alternatively, it might be useful to consider free tortile monoidal categories with idempotent balanced monoidal comonads.

As a second suggestion, which is related to the previous one, but is less ambitious, it should be useful to develop a syntax for traced monoidal closed categories. Perhaps this can be done by extending existing term calculi or proof nets for linear logic.

Finally, useful ways of constructing traced monoidal closed categories should be studied. We have yet to see if the constructions based on uniformity (Hasegawa 2004) can also be used for the closed setting. 


\section{Appendix A. Derivation of sliding and vanishing}

Proposition A.1. Sliding $\operatorname{Tr}_{A, B}^{X}\left(\left(i d_{B} \otimes h\right) \circ f\right)=\operatorname{Tr}_{A, B}^{Y}\left(f \circ\left(i d_{A} \otimes h\right)\right)$ is derivable.

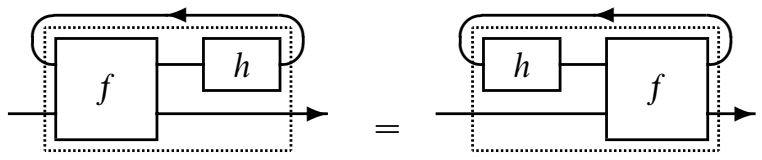

Proof. By Yanking, the left-hand side is equal to

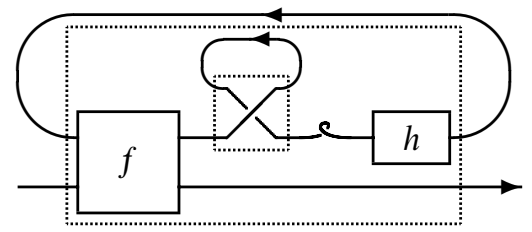

Using Superposing and Tightening, we have

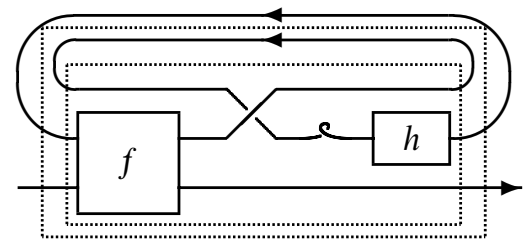

Then we apply Exchange:

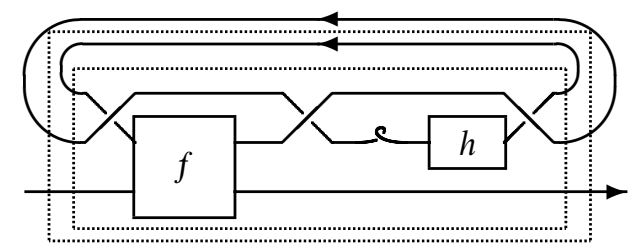

Thanks to the naturality of braidings, this is equal to

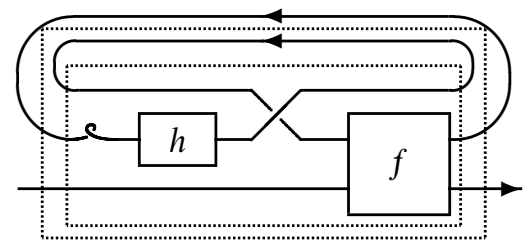

Applying Tightening and Superposing, we obtain

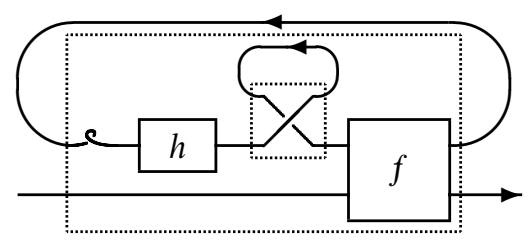

\section{CAmbridge JUUANALS}


By Yanking,

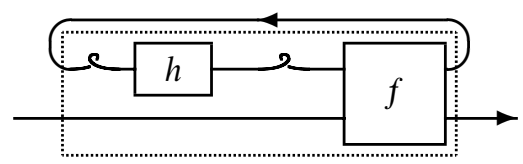

This is equal to the right-hand side of the equation, by the naturality of twisting.

Proposition A.2. Vanishing for tensor $\operatorname{Tr}_{A, B}^{X}\left(\operatorname{Tr}_{A \otimes X, B \otimes X}^{Y}(f)\right)=\operatorname{Tr}_{A, B}^{X \otimes Y}(f)$ is derivable.

Proof. We first show a variant of sliding

$$
\operatorname{Tr}_{A, B}^{X}\left(\operatorname{Tr}_{A \otimes X, B \otimes X}^{Y}\left(\left(i d_{B} \otimes h\right) \circ f\right)\right)=\operatorname{Tr}_{A, B}^{Z}\left(f \circ\left(i d_{A} \otimes h\right)\right)
$$

for $f: A \otimes X \otimes Y \rightarrow B \otimes Z$ and $h: Z \rightarrow X \otimes Y$

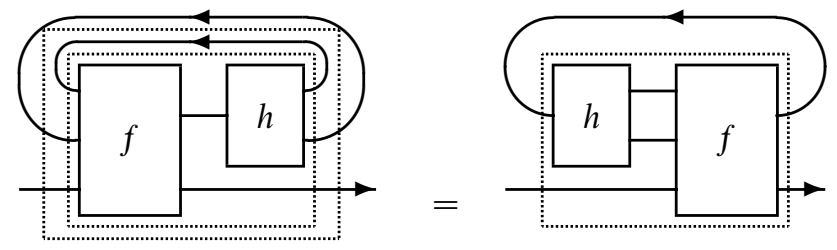

by the same technique as we used for deriving the sliding axiom above. From the left-hand side of the equation, by yanking, superposing and tightening, we get

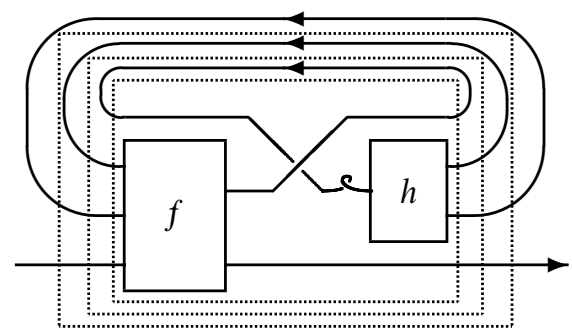

to which we apply the exchange axiom twice:

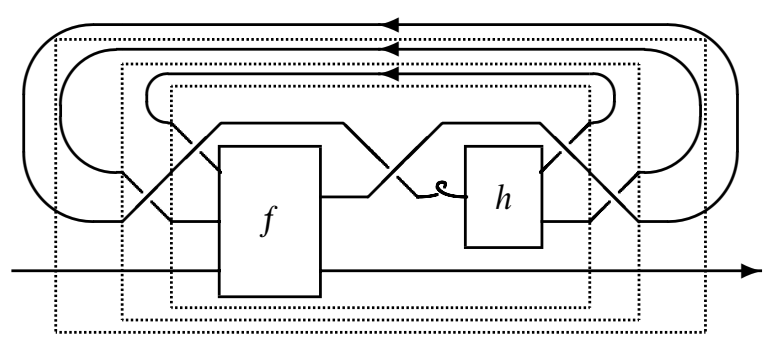

\section{CAMBridge JUURNALS}


By the naturality and bilinearity of braidings, and by tightening and superposing, we have

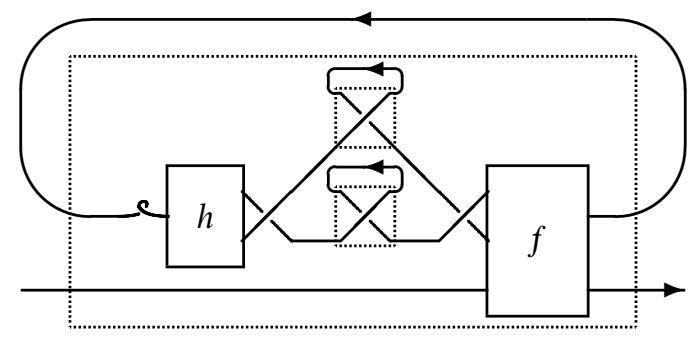

The rest of proof is now easy, by yanking and the naturality of twisting; here we should recall the axiom $\theta_{A \otimes B}=c_{B, A} \circ\left(\theta_{B} \otimes \theta_{A}\right) \circ c_{A, B}$ of twists. The vanishing axiom for tensor follows from this equation by letting $Z$ be $X \otimes Y$ and $h$ be $i d_{X \otimes Y}$.

Remark A.1. Vanishing for unit $\operatorname{Tr}_{A, B}^{I} f=r_{B} \circ f \circ r_{A}^{-1}(f: A \otimes I \rightarrow B \otimes I)$ is redundant in original axioms in (Joyal et al. 1996). For completeness, here is a direct demonstration:

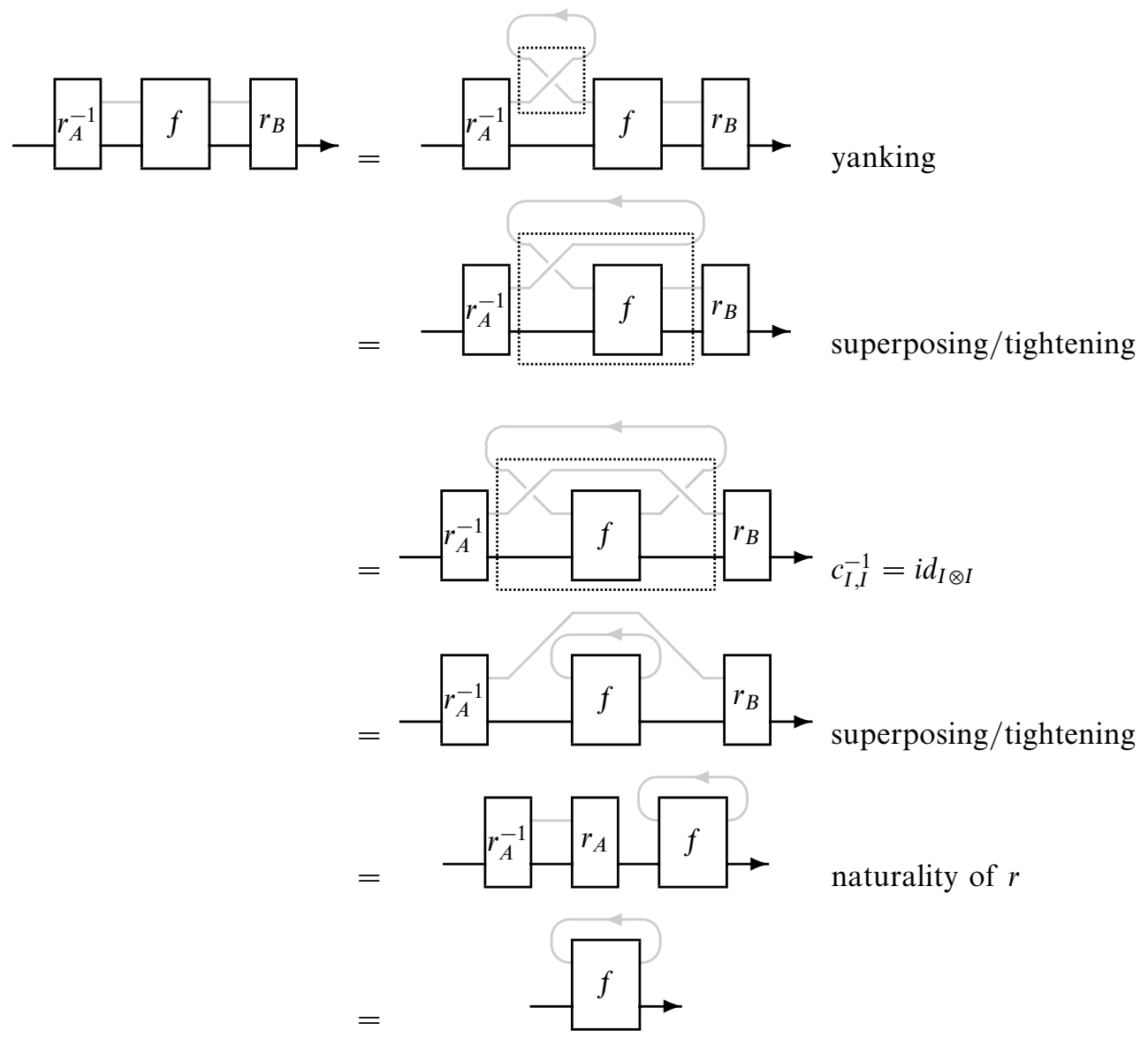




\section{Appendix B. Uniqueness of trace on tortile monoidal categories}

In Section 3.2 we recalled that every tortile monoidal category has a trace called the canonical trace (Joyal et al. 1996). In fact there is no other way of giving a trace to a tortile monoidal category.

Proposition B.1. Suppose that $\mathcal{C}$ is a tortile monoidal category. Then $\mathcal{C}$ has a unique trace. That is, if $\operatorname{Tr}$ is a trace on $\mathcal{C}$, it agrees with the canonical trace, that is,

$$
\operatorname{Tr}_{A, B}^{X} f=\left(i d_{B} \otimes\left(\varepsilon_{X} \circ\left(i d_{X^{*}} \otimes \theta_{X}\right) \circ c_{X, X^{*}}\right)\right) \circ\left(f \otimes i d_{X^{*}}\right) \circ\left(1_{A} \otimes \eta_{X}\right): A \rightarrow B
$$

holds for $f: A \otimes X \rightarrow B \otimes X$.

Proof.

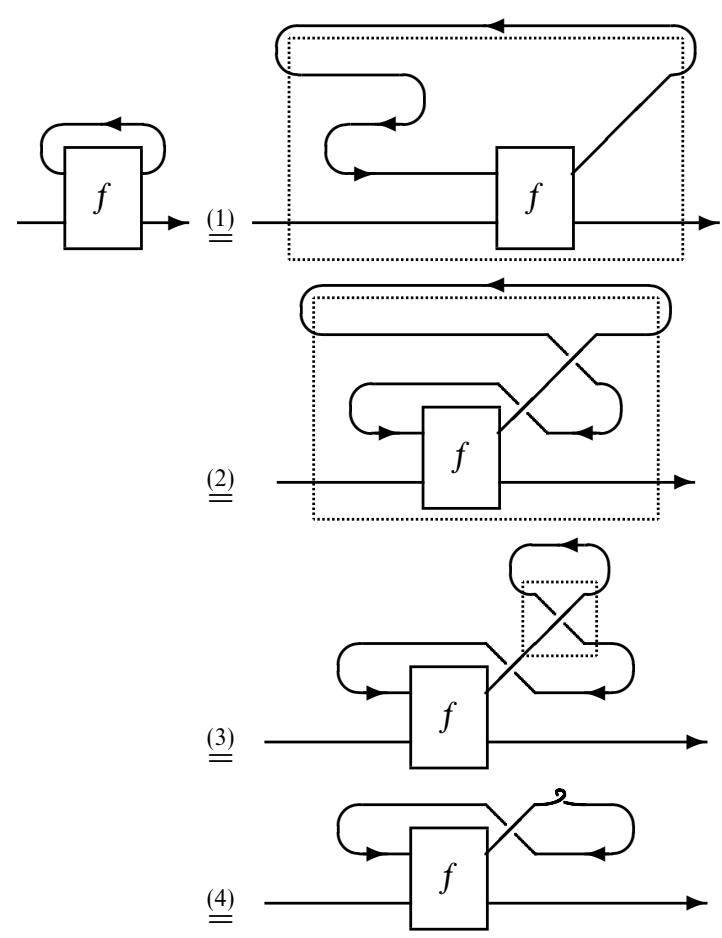

where (1) by an axiom of tortile categories, (2) by naturality and bilinearity of braidings, (3) by tightening and superposing, and (4) by yanking.

\section{Appendix C. Linear fixed-points graphically}

We show that for $f: F A \otimes X \rightarrow X$,

$$
f^{\dagger}=\varepsilon \circ \operatorname{Tr}^{F U X}\left(m^{F^{-1}} \circ F\left(\Delta \circ U f \circ m^{U} \circ(\eta \times i d)\right) \circ m^{F}\right): F A \rightarrow X
$$


satisfies the (linear) fixed-point equation using the box notation for monoidal functors (Cockett and Seely 1999; Melliès 2006):

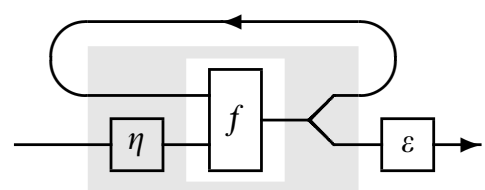

First, duplicate $U f \circ m^{U} \circ(\eta \times i d)$ :

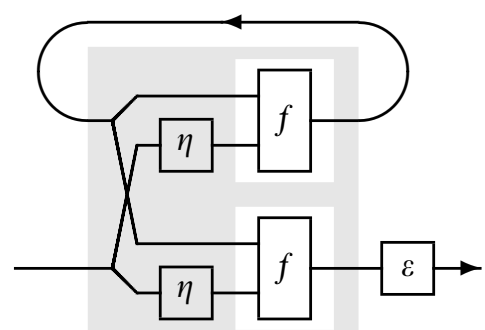

Since $F$ is strong monoidal, the grey box can be decomposed:

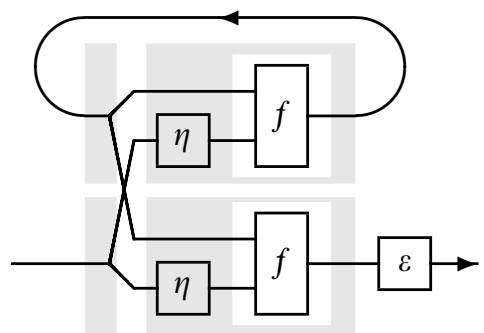

And, furthermore,

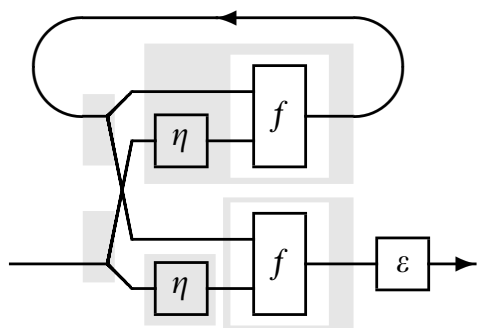

\section{CAmbridge JDUANALS}


Since $\varepsilon$ is a monoidal natural transformation from $F U$ to the identity functor, we have

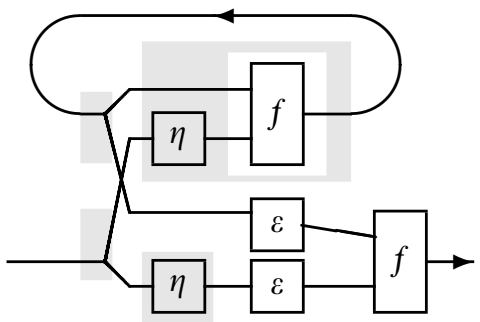

By adjointness, $\varepsilon \circ U \eta=i d$ :

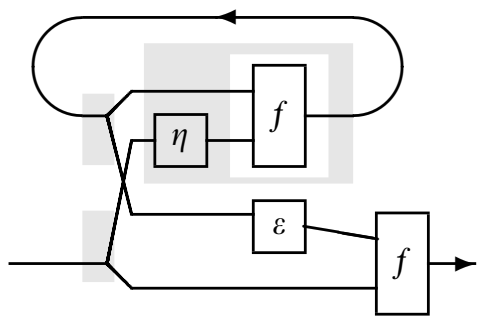

By the axioms of trace,

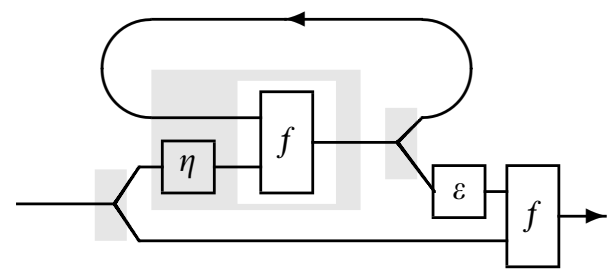

Now we are done: $f^{\dagger}=f \circ(i d \otimes f) \circ m^{-1} \circ F \Delta$,

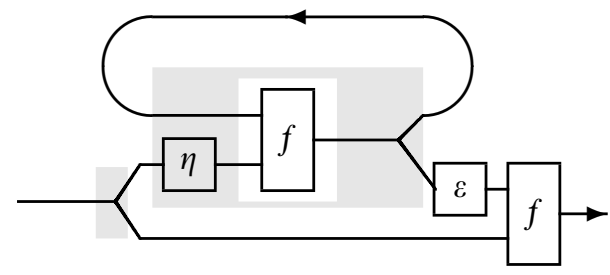

\section{Acknowledgements}

I am grateful to Paul-André Melliès and Shin-ya Katsumata for stimulating discussions and encouragement. Thanks are also due to the anonymous reviewers and to Phil Scott for their helpful comments on the manuscript. This paper is based on a talk given at the workshop on Traced Monoidal Categories, Network Algebras and Applications (TMCNAA) held at Wroclaw on July 2007. I thank the organisers and participants of 
the workshop for much helpful feedback. This work is partly supported by the Japanese Ministry of Education, Culture, Sports, Science and Technology, Grant-in-Aid for Young Scientists (B) 17700013.

\section{References}

Abramsky, S. (1996) Retracing some paths in process algebra. In: Proc. Concurrency Theory. Springer-Verlag Lecture Notes in Computer Science 1119 1-17.

Abramsky, S., Haghverdi, E. and Scott, P. J. (2002) Geometry of Interaction and linear combinatory algebras. Mathematical Structures in Computer Science 12 (5) 625-665.

Abramsky, S. and Jagadeesan, R. (1994) New foundations for the Geometry of Interaction. Inf. Comput. 111 (1) 53-119.

Barber, A. and Plotkin, G. (1997) Dual intuitionistic linear logic (manuscript). An earlier version available as Technical Report ECS-LFCS-96-347, LFCS, University of Edinburgh.

Barr, M. (1991) *-autonomous categories and linear logic. Mathematical Structures in Computer Science 1 159-178.

Benton, N. (1995) A mixed linear non-linear logic: proofs, terms and models. In: Proc. Computer Science Logic. Springer-Verlag Lecture Notes in Computer Science 933 121-135.

Bierman, G. M. (1995) What is a categorical model of intuitionistic linear logic? In: Proc. Typed Lambda Calculi and Applications. Springer-Verlag Lecture Notes in Computer Science 902 78-93.

Bloom, S. and Ésik, Z. (1993) Iteration Theories, EATCS Monographs on Theoretical Computer Science, Springer-Verlag.

Blute, R. F., Cockett, J. R. B. and Seely, R. A. G. (2000) Feedback for linearly distributive categories: traces and fixpoints. J. Pure Appl. Algebra 154 27-69.

Chirica, L. M. and Martin, D.F. (1979) An order-algebraic definition of Knuthian semantics. Mathematical Systems Theory 13 1-27.

Coccia, M., Gadducci, F. and Montanari, U. (2002) GS- $\Lambda$ theories: a syntax for higher-order graphs. In: Proc. Category Theory and Computer Science. Electronic Notes in Theoretical Computer Science 69.

Cockett, J. R. B. and Seely, R. A. G. (1999) Linearly distributive functors. J. Pure Appl. Algebra 143 155-203.

Freedman, M.H., Kitaev, A. and Wang, Z. (2002) Simulation of topological field theories by quantum computers. Commun. Math. Phys. 227 587-603.

Girard, J.-Y. (1987) Linear logic.Theoretical Computer Science 50 1-102.

Girard, J.-Y. (1989) Geometry of Interaction I: interpretation of system F. In: Proc. Logic Colloquium '88 221-260.

Haghverdi, E. (2000) A Categorical Approach to Linear Logic, Geometry of Interaction and Full Completeness, Ph.D. thesis, University of Ottawa.

Haghverdi, E. and Scott, P. J. (2006) A categorical model for the geometry of interaction. Theoretical Computer Science 350 (2-3) 252-274.

Hasegawa, M. (1997) Recursion from cyclic sharing: traced monoidal categories and models of cyclic lambda calculi. In: Proc. Typed Lambda Calculi and Applications. Springer-Verlag Lecture Notes in Computer Science 1210 196-213.

Hasegawa, M. (1999) Models of Sharing Graphs: A Categorical Semantics of let and letrec, Distinguished Dissertations Series, Springer-Verlag.

Hasegawa, M. (2000) A short proof of the uniqueness of trace on tortile categories (manuscript, available from the author's web page). 
Hasegawa, M. (2004) The uniformity principle on traced monoidal categories. Publ. Res. Inst. Math. Sci. 40 (3) 991-1014.

Houston, R. (2008) Finite products are biproducts in a compact closed category. J. Pure Appl. Algebra 212 394-400.

Hyland, M. and Schalk, A. (2003) Glueing and orthogonality for models of linear logic. Theoretical Computer Science 294 (1-2) 183-231.

Joyal, A. and Street, R. (1991) The geometry of tensor calculus, I. Adv. Math. 88 55-113.

Joyal, A. and Street, R. (1993) Braided tensor categories. Adv. Math. 102 20-78.

Joyal, A., Street, R. and Verity, D. (1996) Traced monoidal categories. Math. Proc. Cambridge Phils. Soc. 119 447-468.

Katsumata, S. (2008) Attribute grammars and categorical semantics. In: Proc. International Colloquium on Automata, Languages and Programming. Springer-Verlag Lecture Notes in Computer Science. 5126 271-282.

Katsumata, S. and Nishimura, S. (2006) Algebraic fusion of functions with an accumulating parameter and its improvement. In: Proc. International Conference on Functional Programming 227-238.

Kelly, G. M. (1974) Doctrinal adjunction. In: Proc. Sydney Category Theory Seminar. SpringerVerlag Lecture Notes in Computer Science 420 257-280.

Kelly, G. M. and Laplaza, M.L. (1980) Coherence for compact closed categories. J. Pure Appl. Algebra 19 193-213.

Knuth, D. E. (1968) Semantics of context-free languages. Mathematical Systems Theory 2 (2) 127-145.

Lambek, J. and Scott, P. J. (1986) Introduction to Higher Order Categorical Logic, Cambridge University Press.

Mac Lane, S. (1971) Categories for the Working Mathematician, Graduate Texts in Mathematics 5, Springer-Verlag.

Melliès, P.-A. (2003) Categorical models of linear logic revisited. To appear in Theoretical Computer Science.

Melliès, P.-A. (2004) Asynchronous games 3: an innocent model of linear logic. In: Proc. Category Theory and Computer Science. Electronic Notes in Theoretical Computer Science 122 171-192.

Melliès, P.-A. (2006) Functorial boxes in string diagrams. In: Proc. Computer Science Logic. Springer-Verlag Lecture Notes in Computer Science 4207 1-30.

Melliès, P.-A. and Tabareau, N. (2007) Resource modalities in game semantics. In: Proc. 22nd Logic in Computer Science 389-398.

Milner, R. (1994) Action calculi V: reflexive molecular forms (with appendix by O.Jensen). Manuscript, LFCS, University of Edinburgh.

Penrose, R. and Rindler, R. (1984) Spinors and Space-Time, Vol. 1, Cambridge University Press.

Seely, R. A. G. (1989) Linear logic, *-autonomous categories and cofree coalgebras. In: Categories in Computer Science. AMS Contemp. Math. 92 371-389.

Selinger, P. (2007) Dagger compact closed categories and completely positive maps (extended abstract). In: Proc. International Workshop on Quantum Programming Languages. Electronic Notes in Theoretical Computer Science 170 139-163.

Shum, M.-C. (1994) Tortile tensor categories. J. Pure Appl. Algebra 93 57-110.

Simpson, A. (1993) A characterisation of the least-fixed-point operator by dinaturality. Theoretical Computer Science 118 (2) 301-314.

Simpson, A. and Plotkin, G. (2000) Complete axioms for categorical fixed-point operators. In: Proc. 15th Logic in Computer Science 30-41.

Ştefănescu, G. (2000) Network Algebra, Discrete Mathematics and Theoretical Computer Science Series, Springer-Verlag.

Yetter, D. N. (2001) Functorial Knot Theory, World Scientific. 